\title{
Australian black field crickets show changes in neural gene expression associated with socially-induced morphological, life-history, and behavioral plasticity
}

Michael M. Kasumovic ${ }^{1 * \dagger}$, Zhiliang Chen ${ }^{2,3+}$ and Marc R. Wilkins ${ }^{2,3}$

\begin{abstract}
Background: Ecological and evolutionary model organisms have provided extensive insight into the ecological triggers, adaptive benefits, and evolution of life-history driven developmental plasticity. Despite this, we still have a poor understanding of the underlying genetic changes that occur during shifts towards different developmental trajectories. The goal of this study is to determine whether we can identify underlying gene expression patterns that can describe the different life-history trajectories individuals follow in response to social cues of competition. To do this, we use the Australian black field cricket (Teleogryllus commodus), a species with sex-specific developmental trajectories moderated by the density and quality of calls heard during immaturity. In this study, we manipulated the social information males and females could hear by rearing individuals in either calling or silent treatments. We next used RNA-Seq to develop a reference transcriptome to study changes in brain gene expression at two points prior to sexual maturation.

Results: We show accelerated development in both sexes when exposed to calling; changes were also seen in growth, lifespan, and reproductive effort. Functional relationships between genes and phenotypes were apparent from ontological enrichment analysis. We demonstrate that increased investment towards traits such as growth and reproductive effort were often associated with the expression of a greater number of genes with similar effect, thus providing a suite of candidate genes for future research in this and other invertebrate organisms.
\end{abstract}

Conclusions: Our results provide interesting insight into the genomic underpinnings of developmental plasticity and highlight the potential of a genomic exploration of other evolutionary theories such as condition dependence and sex-specific developmental strategies.

Keywords: Teleogryllus commodus, Black field cricket, Developmental plasticity, Sexual selection, Gene expression, Transcriptome analysis, Behaviour

\section{Background}

Developmental plasticity is common in continuously distributed phenotypes [1]. Although plasticity often leads to differences in morphological and/or behavioural traits (e.g., plasticity in response to pond drying; [2]), it is strongly linked to life-history traits driven by differences in development time [3]. It is specifically this life-history

\footnotetext{
* Correspondence: m.kasumovic@unsw.edu.au

${ }^{\dagger}$ Equal contributors

${ }^{1}$ Ecology \& Evolution Research Centre, School of Biological, Earth and Environmental Sciences, UNSW, Sydney, Australia

Full list of author information is available at the end of the article
}

plasticity that is well studied both theoretically and empirically $[1,4]$ in ecological and evolutionary organisms. Decades of research on life-history driven plasticity has resulted in a strong understanding of the ecological triggers [5-8], adaptive benefits [9-11] and factors necessary for the evolution of such developmentally plastic tactics [12-14].

Despite the insight gained by studying life-history driven developmental plasticity in species with continuously distributed phenotypes, such species are often not ideal for the study of the role of genes in plasticity 
because it is difficult to assign continuous phenotypic differences to specific genetic variation. However, if the phenotypic consequences can be classified in a similar manner as to discrete morphs (e.g., horned beetles) [15] or life-history periods (e.g., hymenoptera) [16] it may allow for a clearer perspective on a gene-phenotype correlation [17] and provide insight into the underlying genomic control of developmental plasticity. In this study, we attempt to overcome this problem by using the Australian black field cricket (Teleogryllus commo$d u s)$, a species that is well-described from an ecological and evolutionary perspective, and one where life-history driven plasticity can be categorized and followed through to maturity and death. We hope that exploring a species with a strong ecological understanding of the factors that result in continuous variation in phenotypic traits has the potential to highlight genes that may be important in life-history decisions and sex-specific variation in developmental strategies.

The Australian black field cricket is a well-studied organism with respect to life-history variation, mating strategies, and how selection affects each of these factors [18-22]. Additionally, both male and female T. commodus possess an interesting socially-induced developmental tactic [5]: males and females alter their resource investment and adult behavior depending on the density and rate of calls they hear in the last instar prior to maturity [23]. Males reared in an environment with a greater density of calls mature later and are heavier and larger than when reared under lower calling densities [23]. Males further match their own calling effort to their local competitive context [24], rendering them more competitive in a crowded environment [18]. In contrast, females in a high density environment mature smaller, but develop significantly faster, allowing them to exploit the high density of available males [23]. Females compensate for their smaller size by producing more eggs [23] and are able to make faster mating decisions [24]. This socially-induced developmental tactic [5] thus results in changes in the relationships between morphological, life-history and behavioural traits, associated with differences in development rate.

The aims of this study are: (a) to generate a de novo transcriptome for T. commodus, (b) to examine whether differences in nymphal gene expression can help explain the differences in developmental trajectories and adult behavior, and (c) to identify transcription factors relevant to the developmental trajectories. Identifying transcription factors in non-model organisms could provide particular insight into important pathways that align with specific life history tactics. One problem in identifying such transcription factors, however, is that they are often expressed in very low rates relative to other genes. We thus explored the expression of transcription factors using self-organised maps (SOM), which are a common bioinformatic technique used in Drosophila organ development [25, 26]. To do this, we reared males and females in two different simulated social environments and examined differences in neural genes expressed between the sexes, in two developmental environments, and in early and late stages of the last juvenile instar prior to maturity. To ensure that we could accurately match the adult morphological, behavioral, and life history traits to the genes expressed, we followed a large number of individuals after maturity until their death. Our results demonstrated that developmental differences correlated with changes in the expression of a small number of genes and transcription factors that regulate maturation, sexual development, and neural development. Moreover, the nymphal alterations in gene expression have lasting effects on adult behaviour and lifespan. We discuss these results with reference to the lifehistory and ecology of the Australian black field cricket.

\section{Methods \\ Cricket rearing}

Outbred recently captured wild type crickets were either 4th (genomics experiment) or 5th (rearing experiment) generation descendants of approximately 300 males and females collected at Smith's Lake, NSW, Australia $\left(32^{\circ} 22^{\prime}\right.$ S, $\left.152^{\circ} 30^{\prime} \mathrm{E}\right)$. We collected nymphs before wing bud formation (which occurs at the penultimate juvenile instar). Each nymph was reared in an individual plastic container $(5 \times$ $5 \times 3 \mathrm{~cm}^{3}$ ) with an egg carton for shelter and supplied with ad libitum food (Friskies Go-Cat senior) and water replaced weekly.

Upon molting to the last juvenile instar, we randomly assigned individuals to either a silent or a low density variable call-quality treatment. Although we have not yet examined the developmental tactic under silence, studies on T. oceanicus (a sister species) demonstrate that males moderate their mating strategies and sperm investment [27], while females moderate their mate preferences [28] in response to these environments. It is thus likely that these two extreme artificial rearing environments will have an effect in $T$. commodus as well. In the variable calling treatment, one of each of the three speakers (Logitech R-10) played a call from a different male at either the mean population calling rate $(17$ calls per minute), a high calling rate (24.5 calls per minute), or a low calling rate (12.6 calls per minute) [23]. We placed speakers in a one metre diameter circle and ensured that all speakers played calls at an amplitude of $70 \mathrm{~dB}$ at the centre of the circle. We reared individuals in two separate acoustically isolated environments and moved treatments between rooms each day to ensure no room effects. 
For the genomics experiment, individuals were sacrificed and dissected at either 3 (early) or 13 days (late) after their last juvenile molt. We chose the early timepoint to allow for a comparison against the late timepoint, and also chose day 3 to minimize any gene expression differences due to molting to the penultimate juvenile instar. We chose the late timepoint because day 13 is the mean development time prior to maturation for crickets reared under 6 different artificial social environments [23]. Although individuals in the different treatments may be at different developmental stages when sacrificed, it allows us to explore the gene expression changes each individual experiences at the same time point. Furthermore, it would be extremely difficult to control for developmental stage in wild-type crickets. This allowed us to investigate whether gene expression differences exists between the two treatments at a point close to molting. We reared a total of 24 penultimate instar nymphs (12 male and 12 female) in two calling treatments (silent and low density-variable quality) and sacrificed individuals at two stages (early or late). This created a balanced design of three individuals (biological replicates) of each sex in each treatment in each time.

We reared another 701 crickets to sexual maturity as part of another larger experiment. For these individuals, we recorded their weight and size (pronotum width) at their final juvenile and adult instars within $24 \mathrm{~h}$ of molting into each instar. This allowed us to calculate their investment into adult size and weight while controlling for the initial starting value as [value at the juvenile instar - value at the adult instar] / value at the juvenile instar; we used these values in our statistical analyses below. After maturity, males were placed in an electronic recording device (callbox) to monitor their calling effort once a week [29]. Briefly, the callbox consists of 256 microphones attached to the lids of the housing containers which are connected to a data logger and personal computer. The computer is programmed to check for a signal from each microphone 10 times per second. The signal is recorded as 1 when $10 \mathrm{~dB}$ higher than the level of background noise, otherwise as 0 . Calling effort is thus counted as the number of seconds a male is heard calling. Females were given a petri dish full of sand as a laying substrate to allow for the separation and counting of eggs.

\section{Statistics}

We used a two-way ANOVA to examine whether there was a sex-specific effect of treatment on the investment into adult size and weight. We also examined whether there were any effects of treatment as a function of sex on development rate $\left(\right.$ days $\left.^{-1}\right)$ and lifespan. We also examined whether there was an effect of treatment on adult reproductive effort, as average nightly calling effort in males and lifetime egg output in females using a GLM with a Poisson distribution and a log link. A proportion of individuals neither called nor produced eggs during their lifetime. Since there was no significant difference in the number of males (calling $=37$, silent $=$ 27; $X^{2}=1.18, \mathrm{P}=0.18$ ) or females (calling $=19$, silent $=$ 23; $\left.\chi^{2}=0.54, \quad \mathrm{P}=0.46\right)$ that were not reproductively active between the treatments, we removed these individuals from our analyses.

\section{Dissections and extractions}

We anesthetized Individuals on dry ice for two minutes prior to dissections. All brain dissections were performed in $0.01 \mathrm{M}$ phosphate-buffered saline containing $3 \%$ Triton X-100 on a bed of dry ice and completed within two minutes. We minimized temporal variation in gene expression by performing dissections between 1-2 pm each day. Upon completion of dissections, brains were immediately stored in a $-80{ }^{\circ} \mathrm{C}$ freezer until extraction a maximum of 10 days later. We used a QIAGEN RNeasy Plus Universal Tissue Mini Kit for RNA extractions, following the manufacturer's protocol.

\section{Library preparation and transcriptome sequencing}

Brain tissue of 12 males and 12 females, equally from each rearing treatment (Silent, Calling) at two time points (Early: day 3, Late: day 13) were used for the isolation of mRNA using the Isolate II RNA Mini Kit (Bioline). The cDNA libraries for Illumina HiSeq 2000 sequencing were constructed from $10 \mu \mathrm{g}$ of total RNA from each brain using the Illumina TruSeq RNA Sample Prep Kit (version 2) according to the manufacturer's instructions. Equal amounts of total RNA from each sample were barcoded separately $(n=24)$ after prep to allow for multiplexing in a single lane. Each lane containing the eight multiplexed libraries had an equal distribution of sexes, treatments, and timepoints to control for bias. Libraries were then sequenced on the HiSeq 2000 using TruSeq v3 SBS reagents to generate $101 \mathrm{bp}$ paired-end reads with an approximate insert size of $160 \mathrm{bp}$, following the standard Illumina protocol. This resulted in an average of 80 million paired-end reads per individual. All sequencing was completed in the Ramaciotti Centre for Genomics, the University of New South Wales.

\section{De novo assembly of cricket transcriptome}

Prior to RNA-Seq analysis, filters were applied to remove low quality reads from all twenty-four paired-end samples. Initial quality assessment for Illumina HiSeq sequence data was based on FastQC (version 0.11.2) (http://www.bioinformatics.babraham.ac.uk/projects/ fastqc/) [30] statistics, and Cutadapt (version 1.2.1) [31] was used for adapter/primer trimming. We then trimmed paired-end raw reads with the BWA trimming 
mode at a threshold of Q13 $(\mathrm{P}=0.05)$ as implemented by SolexaQA version 1.11 [32]. Low-quality 3' ends of each read were filtered. Reads that were less than $25 \mathrm{bp}$ in length were discarded.

RNA-Seq reads from 8 individuals containing each of the sex, treatment, and age conditions sequenced in the same Illumina lane were selected for assembly. This resulted in a total of 489.7 million $101 \mathrm{bp}$ paired-end reads, that after trimming and filtering for quality and length respectively, gave 473.2 million PE reads. Transcriptome short reads were assembled de novo by ABySS then Trans-ABySS [33], Velvet-Oases [34] and Trinity [35]. The workflow for the transcriptome assembly, evaluation and annotation is summarised in Additional file 1: Figure $\mathrm{S} 2$ and greater details about the assembly and assemblers can be found in Additional file 2 : Supplementary Materials and Results.

The three assembled transcriptomes were compared by total size, N50 and sequence coverage. The Oases-assembled transcriptome had a total size of 199,904,425 bp made of 80,476 transcripts; this was the highest among the three assemblers. The Oases assembly also had the highest percentage of contigs covered by the other two assemblies (Additional file 1: Table S1). Reads from 24 individual samples were aligned to the three assemblies using Bowtie2 version 2-2.0.0-beta7 with the default parameters. The percentage alignment rates were calculated by Bowtie2 [36]. Manipulating alignment results involved the use of SAMtools version 0.1.18 [37]. The Oases assembly had the highest percentage of reads able to be mapped to the assembled transcriptome by Bowtie using default parameters; this was $0.5 \%$ higher than the Trans-ABySS assembly, and $20 \%$ higher than the Trinity assembly (Additional file 1: Table S1). Accordingly, the Oases-assembled transcriptome was selected as the candidate for further analyses.

We next evaluated the assembled transcriptome by comparing it to available reference transcriptomes to evaluate the quality of the de novo assembly results. The closest related species was the sister species, T. oceanicus. We obtained 41,962 de novo assembled $T$. oceanicus transcripts [38] and selected 32,643 transcripts of lengths greater than $200 \mathrm{bp}$ for the comparison. A total of 50,945 T. commodus transcripts assembled by Oases had BLASTN hits to the 12,959 transcripts in the $T$. oceanicus transcriptome, of which 36,411 hits we re of high quality. We defined high quality hits as a minimum of $80 \%$ alignment length of the pair of sequences and where the percentage identity is equal or greater than $80 \%$ in the alignment as $80 \%$ sequence similarity provides strong evidence of identifying two transcript sequences from similar species with similar function [39]. Among the high quality alignments, the average sequence similarity was $98.5 \%$ and the average T.oceanicus transcript length coverage was $97.2 \%$. Although there is no gold standard for assessing transcriptome quality, these comparisons show that the assembled T.commodus transcriptome is at least comparable to the published T.oceanicus transcriptome. The T.commodus assembled transcriptome for all samples was submitted to the Transcriptome Shotgun Assembly (TSA) at NCBI; the details can be found at the end of the manuscript.

Despite the opportunity for comparison, the T. oceanicus transcriptome may not be a complete transcript set and not well annotated. We therefore decided to further validate our T. commodus transcriptome by performing a BLAST search against the complete set of transcripts of Drosophila melanogaster from FlyBase [40]. As the comparison is based on ortholog level, high quality hits were defined by a different rule as compared to the rule used in the $T$. oceanicus comparison. High quality hits were defined as a minimum of $80 \%$ of the length of the reference sequence and a minimum of $50 \%$ percentage identity in the alignment. A total of 47,763 hits to the $D$. melanogaster genome were identified by a BLAST search, of which 11,768 were high quality hits.

\section{Functional annotation and classification}

To functionally annotate the cricket transcriptome, the final assembled transcripts ( $\geq 200 \mathrm{bp}$ ) were submitted for homology and annotation searches using Blast2GO software (version 2.4.4; https://www.blast2go.com/). For BLASTX against the NR database, the threshold was set to E-value $\leq 10^{-6}$. GO classification was achieved using WEGO software [41]. Enzyme codes were extracted and Kyoto Encyclopedia of Genes and Genomes (KEGG) [42] pathways were retrieved from the KEGG web server (http://www.genome.jp/kegg/).

Using BLAST2GO (version 2.4.4), we were able to assign gene annotations to 46,774 of the 80,476 transcripts from the Oases assembly. Gene ontologies (GOs) were also assigned to the assembled transcripts by BLAST2GO. There were a total of 90,357 gene ontology (GO) terms on all GO-levels associated with the 46,774 identified genes. Of these, assignments to level two GO-terms Molecular Function (40,244) made up the highest category, followed by Biological Process $(33,225)$ and Cellular Components $(16,888)$.

\section{Mapping of RNA-Seq and differential expression analysis} Gene expression levels were determined by quantifying the observed read abundance. As RNA-Seq reads can be mapped to multiple genes or isoforms, we used a read mapper capable of fully handling reads that map ambiguously between both isoforms and genes. We used the RNA-Seq by Expectation-Maximization (RSEM) package version 1.2.0 [43] with default settings to resolve ambiguous mappings and to perform final quantifications when assigning reads to genes and isoforms and counting transcript abundances. In each pair-wise comparison, we identified the significantly differentially 
expressed genes using the edgeR package [44], using the normalized read counts provided by RSEM. Although reference-based transcriptome analysis normally uses a $\mathrm{q}$ value $<0.1$ and Benjamini and Hochberg FDR $<0.05$, due to the nature of transcriptome assembly, the assembled transcriptome may have included a higher number of duplicated transcripts which would affect the read mapping and counting. Thus, to avoid missing any false negative values, we have chosen less stringent values for Benjamini and Hochberg FDR (10 \%) and p value (0.05) for our analysis. We also provide a zip file with the expression values of the transcripts and their corresponding TSA transcript IDs to allow examination of the differentially expressed T. commodus genes in Additional file 3.

\section{Functional analysis of gene lists using DAVID}

The Database for Annotation, Visualization and Integrated Discovery (DAVID) v6.7 is a set of web-based functional annotation tools [45]. The functional clustering tool was used to look for functional enrichment for corresponding Drosophila genes differentially-expressed in each condition. A unique list of gene symbols was uploaded via the web interface, and the background was selected as Drosophila melanogaster. We selected the Biological Process Gene Ontology as the functional annotation category for this analysis.

\section{Extraction of transcription factors}

We downloaded a curated list of candidate Drosophila transcription factors identified on the basis of a structural domain assignment (for a DNA-binding domain) or previous Gene Ontology annotation for a transcription factor related term from the Drosophila Transcription Factor Database (v2.0) [46]. We used these transcription factor sequences as the queries for searching transcription factor sequences in our assembled cricket transcriptome. From the assembled transcriptome, 3,145 transcripts had BLAST hits to the D. melanogaster transcription factor list. For the transcription factor analysis, a final list of 2,418 transcripts from the T. commodus transcriptome was confirmed by excluding transcripts that had no read mapped in three or more individuals.

\section{Self-Organised Maps (SOMs) for extracting the expression} pattern on Transcription Factors

The implementation of The Kohonen Self-Organizing Feature Map was used to build the SOMs. The average count of each extracted transcription factor from all 3 biological replicates of each condition is then calculated from the count matrix produced by RSEM and a new Average Count Matrix is build using these average counts and is used in the later steps. The average count matrix is then normalized by 'genescale' function in the 'genefilter' Bioconductor package [47] to have a mean of 0 and a standard deviation of 1 . The Kohnonen pakage [48] in $\mathrm{R}$ then uses the normalized average count matrix to generate the SOMs. SOMs can be summarized in any number of grids, however, it is beneficial to choose a grid size that visually presents the gene expression patterns as clear separations in a distinguishable way. As a result, we trialed several different grid sizes and settled on a 5-by-5 grid as this provided the best visual separation of gene expression differences. The $\mathrm{R}$ scripts for generating the SOMs can be found at https://github.com/ latrodektus/cricket_genomics.git.

\section{Results and Discussion}

\section{Morphology, life-history, and behaviour}

Crickets were reared in two treatments; one was silent, the other where crickets were exposed to frequent, recorded calling. A total of 352 females (calling $=178$, silent $=174)$ and 349 males $($ calling $=179$, silent $=170)$ were approximately equally divided between the two treatments. As seen in our previous studies [23, 24, 49], there was a significant effect of treatment on the sexspecific expression of life-history, behavioural, and reproductive traits. Although there was no effect of treatment on either the investment towards body size or weight (Table 1), both males and females matured more quickly in the silent compared to the calling treatment (Table 1, Fig. 1). This treatment effect, however, is driven by females (Tukey HSD $=0.05$ ) since there was no difference in the development rate between males in the two treatments (Tukey HSD $=0.74$ ). There was also a difference in development between the sexes. Females developed

Table 1 The effect of treatment and sex on four life-history traits

\begin{tabular}{llll}
\hline & $F$ & d.f. & $P$ \\
\hline Size increase & & & \\
Sex & 4.95 & 1,697 & 0.03 \\
Treatment & 0.20 & 1,697 & 0.65 \\
Sex $\times$ Treatment & 0.60 & 1,697 & 0.44 \\
Weight increase & & & \\
Sex & 4.95 & 1,697 & 0.02 \\
Treatment & 0.20 & 1,697 & 0.65 \\
Sex $\times$ Treatment & 0.60 & 1,697 & 0.44 \\
Development rate & & & \\
Sex & 41.13 & 1,697 & $<0.0001$ \\
Treatment & 6.46 & 1,697 & 0.01 \\
Sex $\times$ Treatment & 1.19 & 1,697 & 0.28 \\
Lifespan & & & \\
Sex & 22.55 & 1,697 & $<0.0001$ \\
Treatment & 0.24 & 1,697 & 0.62 \\
Sex $\times$ Treatment & 6.76 & 1,697 & 0.009 \\
\hline
\end{tabular}



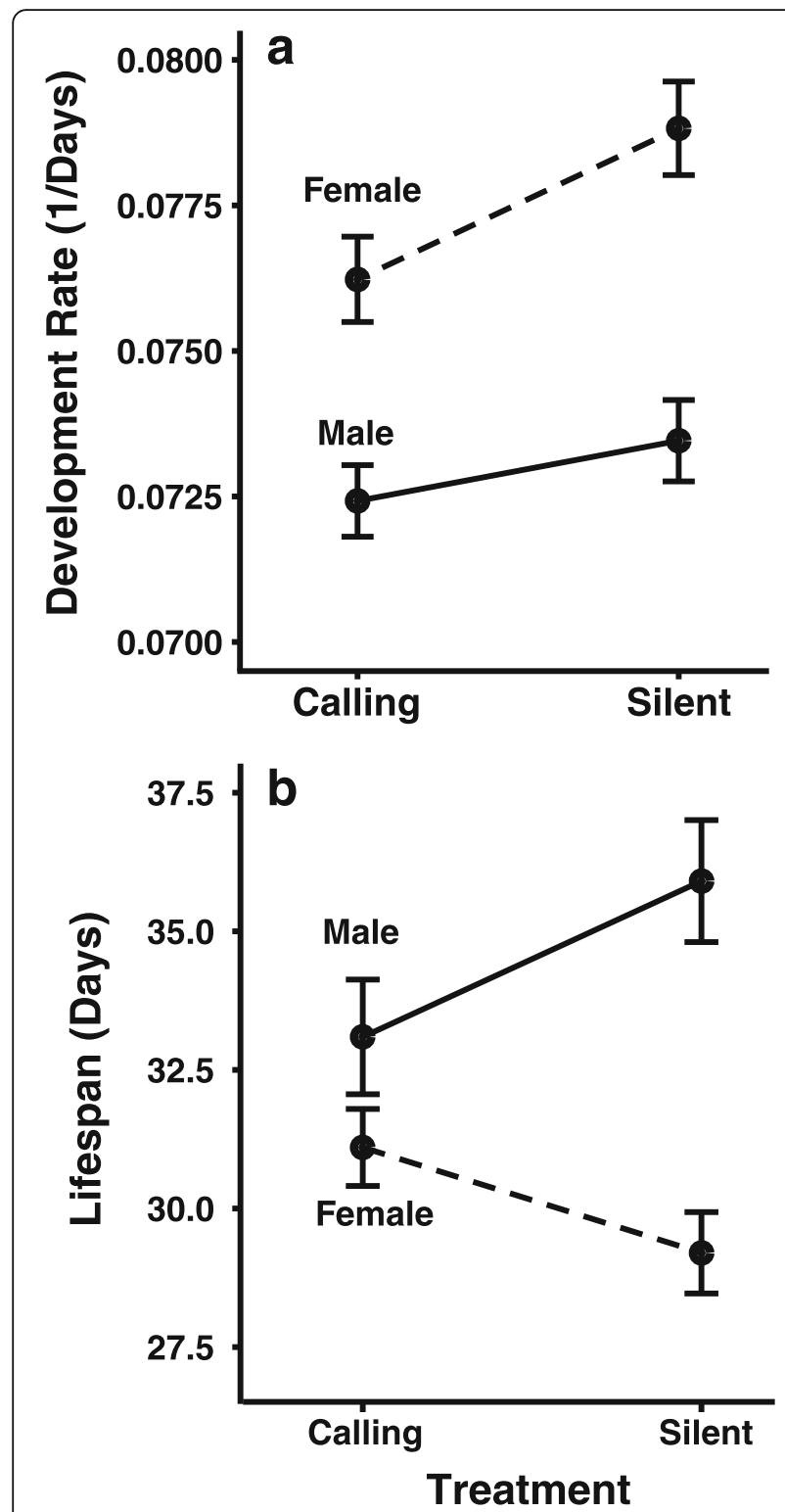

Fig. 1 The difference in the developmental rate (a) and lifespan (b) of males and females reared in the silent and calling treatments. Bars are standard errors

faster than males (Table 1, Fig. 1), and also invested relatively more resources towards size (controlled for penultimate size; female: $0.156 \pm 0.002 \mathrm{~mm}$, male: $0.149 \pm$ $0.002 \mathrm{~mm}$ ) and weight (controlled for penultimate size; female: $0.665 \pm 0.011 \mathrm{~g}$, male: $0.567 \pm 0.010 \mathrm{~g}$ ) in their final instar relative to males. There was also a sex-specific effect of treatment on lifespan with males generally living longer than females; however, the silent treatment had the opposite effect on the sexes with males and females respectively showing an increase and decrease in lifespan (Table 1, Fig. 1).

As a total of 30 males (calling $=18$, silent $=12$ ) died prior to being placed in the callbox, the analysis for calling effort is based on 319 individuals. The median nightly calling effort of adult males from the calling treatment was lower (1660 calls; 95 \% CI: 3034-4193) than males from the silent treatment (2459 calls; $95 \%$ CI: 2634-3968; $\left.X^{2}=47.36, P<0.0001\right)$. Adult females from the calling treatment had a higher median lifetime egg production (425 eggs; $95 \%$ CI: 408-506) than females from the silent treatment (408 eggs; $95 \% \mathrm{CI}$ : 400-495; $\left.\chi^{2}=47.36, P<0.0001\right)$.

Our results thus replicate [23, 24, 49] and demonstrate four developmental and life-history tactics for which we can explore underlying differences in gene expression. First, as females invest more towards their growth and development rate relative to males (Fig. 1a), we expect to see a relative increased expression of genes associated with development, and maturation compared to males. Males, in contrast, invested significantly more resources towards lifespan (Fig. 1b) and we expect to see a relative increase in the expression of genes involved in life extension compared to females. Given that we also see a sexspecific effect of treatment on life-history and performance traits, we expect differences in sex-specific gene expression as a consequence of treatment. As males in the silent treatment had the longest lifespan and had a higher median-nightly calling effort, we expect a greater relative expression of genes associated with lifespan, energy metabolism, and courtship behavior compared to males in the calling treatment. In contrast, as females demonstrated a significant decrease in lifespan in the silent treatment compared to the calling treatment, accompanied with lower median reproductive effort, we expect a relative decrease in the expression of genes associated with lifespan and reproductive output in the silent treatment.

\section{Age-related gene expression differences}

To assess the gene expression profiles, sequenced reads from all 24 individuals were mapped to a de novo transcriptome, which we assembled with Oases (See Methods). We used RSEM version 1.2.0 with default settings [43] to assign reads to isoforms and to calculate transcript abundance. From all 24 samples, an average of $97 \%$ of reads were mapped to the transcriptome by Bowtie. We provide a heatmap to show the differential gene expression and clustering of samples according to expression values in Fig. 2.

Differential gene expression analysis revealed significant differences in brain gene expression between crickets sacrificed on day 3 and those sacrificed on day 13 (>2-fold in expression and $p$-value $<0.05$ ). Due to the difficulty of analyzing the differential expression of a large number of transcripts $(80,476$ transcripts from 24 individuals) from both sexes between the two treatments at both time periods $(2 \times 2 \times 2)$, we split the data into 


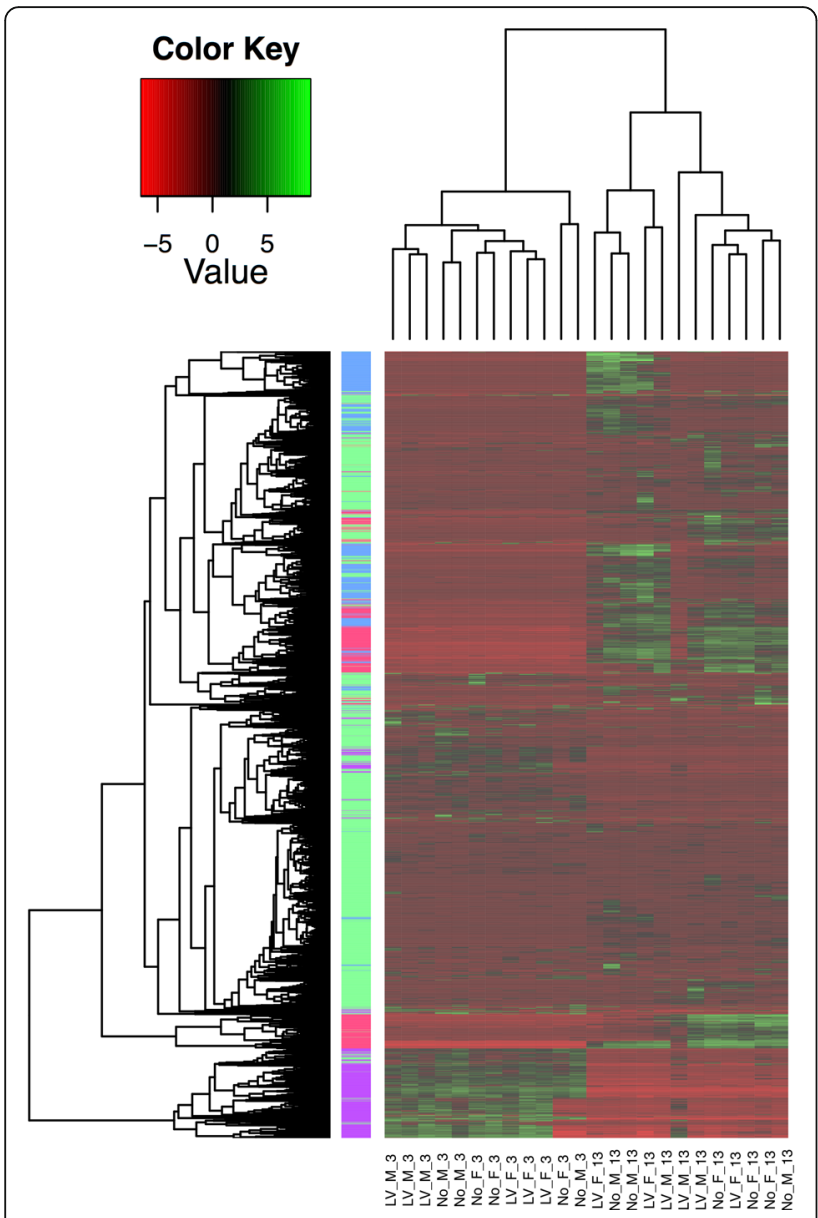

Fig. 2 A heatmap showing the differential gene expression and clustering of samples according to expression values. The samples are on the left axis with notation for the treatments ( $L V=L O W$-Variable calling treatment; $\mathrm{No}=$ Silent Treatment), Sex ( $F=$ Female; $M=$ Male), and age ( $3=3$ Days; $13=13$ Days). The top axis is the gene relationships, and the right axis is the clustering of the samples

two temporal sets of transcripts (day 3 and day 13) as they contained related expression patterns (Additional file 1: Figure S1). In the temporally split sets, there were a total of 6,366 transcripts overexpressed in the brains of crickets sacrificed on day 3 compared to crickets sacrificed on day 13 (3,507 of which were successfully annotated), and 2,266 transcripts overexpressed in the brains of all crickets sacrificed on day 13 compared to all crickets sacrificed on day 3 (1,562 of which were successfully annotated). All the overexpressed transcripts in each time period fell into four Gene Ontology (GO) clusters: the regulation of muscle development, moulting, metabolic processes, and cell development and organization (Fig. 3, Additional file 2: Excel file).

In examining the temporal differences in expressed GO clusters, moulting-related genes accounted for the largest group of genes overexpressed in crickets sacrificed on day 13 compared to those sacrificed at day 3;
341 transcripts were significantly increased in their expression in the crickets sacrificed in day 13 compared to those sacrificed at day 3 , while only 7 moulting related transcripts had significantly greater expression in crickets sacrificed on day 3 compared to those sacrificed on day 13. Crickets sacrificed on day 13 also had greater expression of 12 juvenile hormone esterase or epoxide hydrolase related proteins (proteins that trigger moulting, [50]) compared to those sacrificed on day 3. Only a total of 2 transcripts related to juvenile hormone epoxide hydrolase were found to be overexpressed in the early period, while 28 were found overexpressed in the later period. This difference between the crickets sacrificed on day 3 and 13 is explained by the synthesis, secretion, transport and accumulation of moulting proteins necessary to prepare for the moulting processes that occur closer to maturity. Our expression profiles described here thus seem to accurately describe the developmental progression from metabolic and catabolic processes required during early development, to the genes associated with maturation and moulting later in development.

We were initially surprised to see GO clusters associated with muscle development expressed in the brain, and it is likely that this expression is a result of the contamination from the muscle tissue surrounding the brain. Nonetheless, the pattern of increases in the GO clusters expressed in male crickets from the calling treatment sacrificed on day 13 is interesting as it follows the same pattern as those seen in metabolic processes, and cell development and organization GO clusters. This suggests that future studies focusing specifically on muscle may be interesting.

\section{Treatment and sex related differences}

To delve more deeply into the expression differences between the sexes in each treatment for each time period, we mapped each of the transcripts to known Drosophila genes and focused on exploring the genes associated with biological processes in the behavioural, developmental, and life-history shifts demonstrated by juveniles in this study. As a result, we focused on genes that played roles in growth/maturation, lifespan, mating/ courtship, flight/energy production, spermatogenesis/ oogenesis, aggression, and memory/learning where the function is well documented by either mutant lines or knock-outs.

In day3, we mapped the 200-600 unique transcripts in each treatment by sex combination to 458 unique Drosophila genes. Of these genes, we found 25 genes that were overexpressed by one sex by treatment combination relative to the others, thus being unique to a single sex by treatment combination (Additional file 2: Excel file). In day13, we mapped the 200-600 unique 


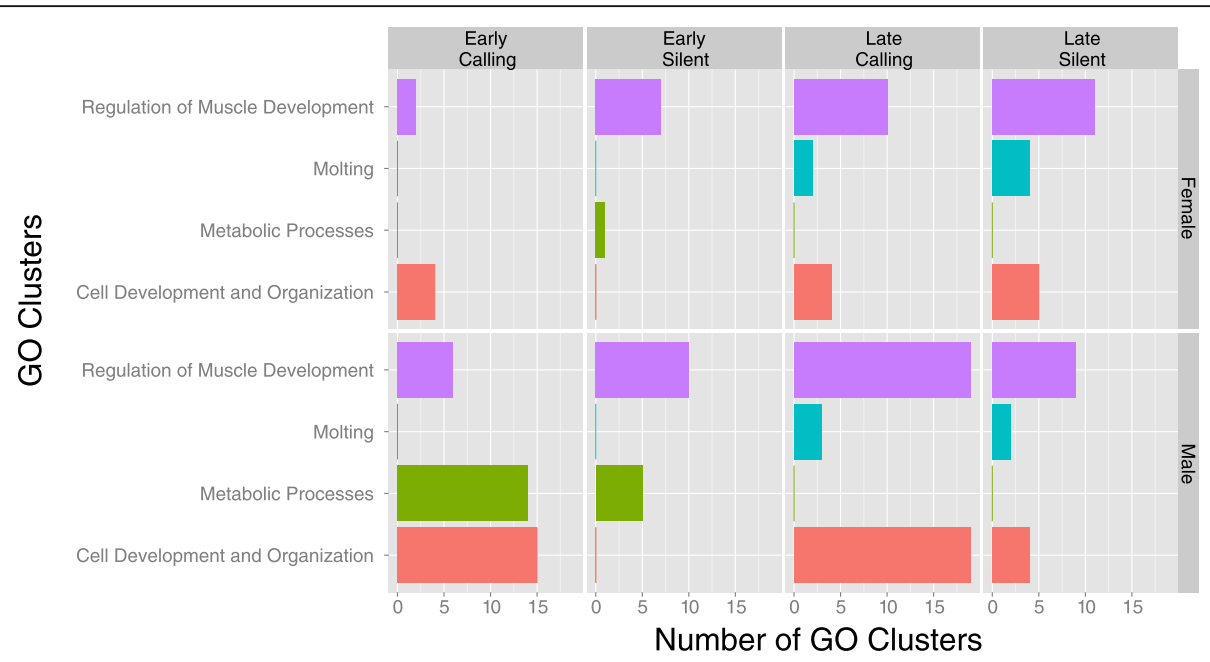

Fig. 3 The number of gene ontology (GO) clusters demonstrated to be overexpressed by comparisons between each sex and each treatment within each time period. The GO clusters listed in the figure are GOs that are associated with the regulation of muscle development, molting, metabolic processes, cell development and organization, and molting in the early and late time periods. Each color represents a specific GO cluster. As comparisons are made within each time period, the bars do not represent relative differences in expression between sexes and treatments between time and thus cannot be compared between time periods

transcripts in each treatment by sex combination to 563 unique Drosophila genes. Using the same procedure as in day 3, we found 21 genes unique to single sex by treatment combinations (Additional file 2 :Excel file). We discuss each of the treatment by sex combinations individually below and provide documented references and FlyBase IDs for each of the genes discussed below in the Additional file 2: Excel file.

\section{Males reared in silence}

Males in the silent treatment lived the longest (Fig. 1) and called the most. The significantly increased lifespan by males was paralleled with significantly higher expression of four separate genes associated with an increase in lifespan (Fig. 4). Males had higher expression of ruby, which directly contributes to increased lifespan [51]. Males also had higher expression of three genes that are known to indirectly positively affect lifespan: (1) puckered, which significantly increases lifespan through a decrease in reactive oxygen species [52, 53], increased immune system function [54], and wound healing [55, 56], (2) p38b MAP kinase which increases lifespan through immune response [57] and responses to ROS [58], and (3) mitochondrial trifunctional protein a subunit associated with increased lifespan through increased storage of lipid concentrations [59] and improved wound healing [60].

The increased calling effort shown by males in the silent treatment was paralleled by the expression of genes positively associated with courtship behavior and energy production, aspects that could result in more efficient calling effort (Fig. 4). All males reared in silence had a greater expression of Neuroglian compared to the other treatments. Neruoglian affects male courtship behavior with male Drosophila with increased expression performing a more intense courtship with higher courtship speeds [61]. This change in Neuroglian was paralleled with increases in genes associated with pathways of greater energy production. The first gene, Glycerol 3 phosphate dehydrogenase, is associated with changes in flight capacity of Drosophila due to increased tryglyceride energy stores [62]. The second, Hyperkinetic, is involved in potassium ion transport and regulates voltage-gated $\mathrm{K}$ channels in muscle fibers making them more efficient [63].

Even though males reared in silence did not mature more quickly than their counterparts reared with recorded calling, males from the silent treatment overexpressed two genes positively associated with moulting, TATA box binding protein-related factor 2 which responds to ecdysone during the onset of moulting [64] and ftz transcription factor 1 which is necessary for proper moulting through activation of ecdysone receptors by juvenile hormone [65]. It is interesting that these genes are expressed in males from the silent treatment as females from the silent treatment matured most quickly (Fig. 1A).

\section{Females reared in silence}

Females reared in silence demonstrated the most growth. Associated with their faster development, these females increased the expression of four genes whose expression is positively associated with growth and development (Fig. 4): (1) bellwether, a gene associated with the translation initiation factor Eif $4 A$ that behaves 


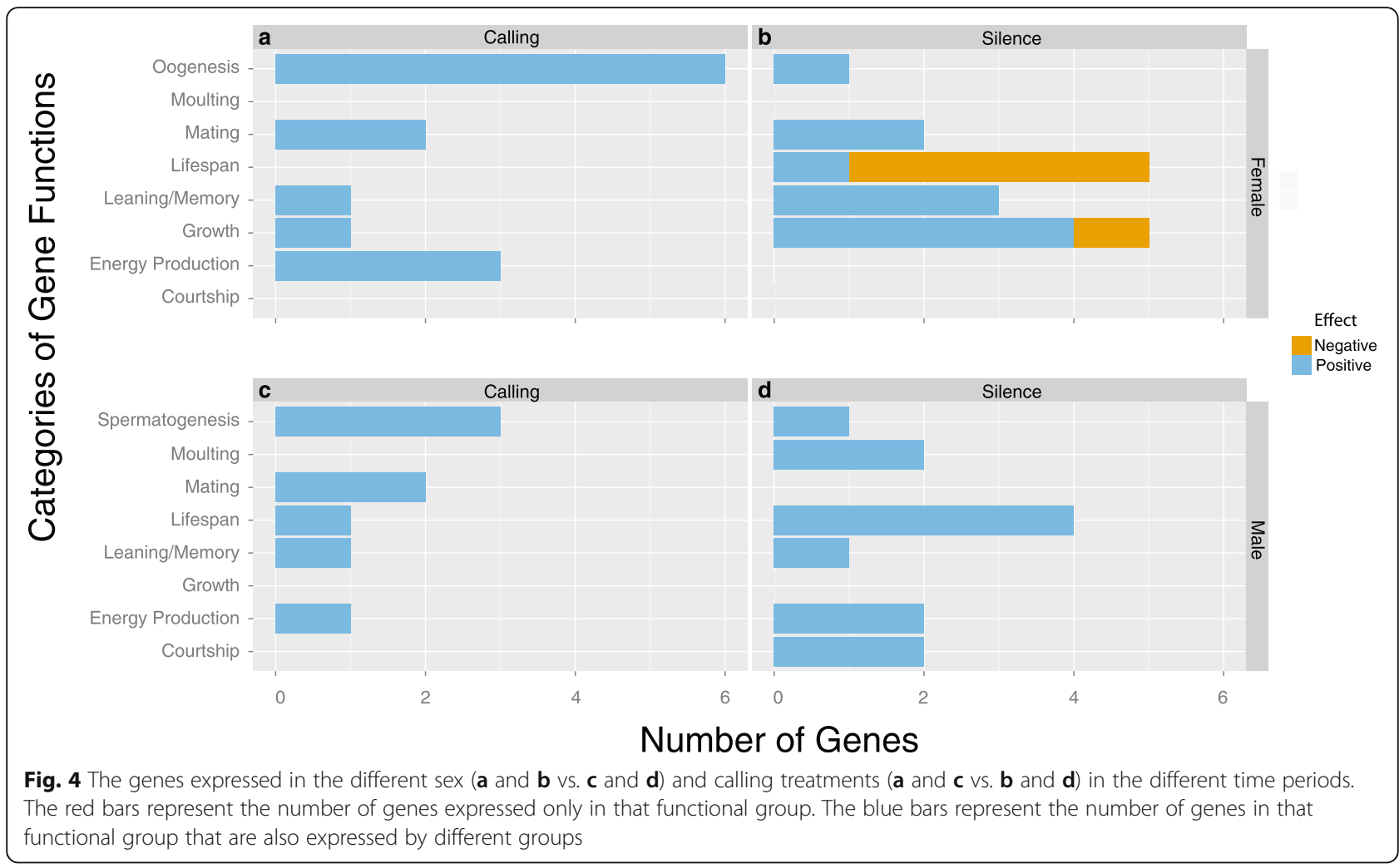

as a dose-dependent growth regulator [66], (2) yorkie, a gene involved in increased growth by positively regulating transcription [67, 68], and (3) Juvenile hormone esterase associated with increased growth [69] and mating [70]. Females also increased expression of slimfast, a gene where non-functioning mutants show growth similar to nutrient starved individuals [71].

Females reared in silence also demonstrated the shortest lifespan compared to all other animals (Fig. 1b). In line with this observation, of the five genes involved in lifespan expressed by females from the silent treatment (Fig. 4), four of the genes are negatively associated with lifespan. Specifically, increased expression of myospheroid directly results in a decreased lifespan [72] and Autophagy-related $8 a$, where suppression of this gene shows increased lifespan [73]. Overexpression of two other genes are known to decrease lifespan through a more indirect route: superoxidase dismutase 2 is associated with reduced lifespan due to the costs of greater oxidative capacity [74] and light which interacts with the gene blue cheese which is associated with a decreased lifespan [51]. Females did, however express a single gene, Neural Lazarillo, where increased activity increases lifespan, but decreases growth [75].

The expression of genes associated with decreased lifespan is particularly interesting as they suggest a costly trade-off where females mature earlier, but live shorter lives. Studies specifically examining this trade-off through selection lines would provide a particularly interesting perspective on the association between development rate and longevity as these traits are shown to trade-off in other studies using T. commodus [76, 77].

Females from the silent treatment only expressed a single gene associated with egg output, midway [78], which may help explain the difference in egg output by females between the two treatments (see below).

\section{Females reared with recorded calls}

Females reared in the calling treatment only showed increased expression of a single gene associated with faster development, myopic, a gene that indirectly affects growth through its interaction with yorkie [79] (Fig. 4). This suggests that yorkie is an important factor in determining growth in $T$. commodus and may explain increased growth of females relative to males (Fig. 1).

Females reared in the calling treatment also produced relatively more eggs than females reared in the silent treatment. As was predicted, these females had higher expression of two unique genes, spinster and tho 2 each of whose expression is associated with increased oogenesis [80, 81]. Four other genes Rab5 [82], stumps [83], RNA-binding protein 9 [84], and COP9 signalosome subunit 8 [85] are associated with germline maintenance necessary for proper oogenesis. Females from the calling treatment, however, also demonstrated increased expression of genes associated with energetic pathways (Fig. 4), 
increasing expression of citrate synthase, a marker of functioning mitochondria [86], thiolase which interacts with mitochondrial trifunctional proteins [59] and $N A D H$ dehydrogenase (ubiquinone) $20 \mathrm{kDa}$ subunit which is involved in the electron transport chain [87].

Although not examined directly in this study, we discuss the gene expression results associated with mating and sexual communication in Additional file 2: Supplementary Materials and Results as these behaviors were examined in previous studies on a sister species, $T$. oceanicus $[27,28]$.

\section{Males reared with recorded calls}

In contrast to males in the silent treatment, males from the calling treatment expressed 6 unique genes, only one of which is positively associated with lifespan (four wheel drive) [88] (Fig. 4). This may explain the increased lifespan relative to females in both treatments, and the relatively decreased lifespan relative to males reared in the silent treatment (Fig. 1b). The other five genes were associated with mating and spermatogenesis (Fig. 4), behaviours that were not specifically examined in this study. However, because mating and spermatogenesis were not the focus of our study, but were examined in a sister-species, T. oceanicus, following a similar protocol [89], we discuss them in greater detail in Additional file 2: Supplemental Materials and Results.

\section{Summary}

Our above results highlight 45 candidate genes (Additional file 2: Excel file) that are associated with various life-history, morphological, and behavioural plasticity in our treatments and that have long been under study in T. commodus and other species. These results are intriguing for two reasons. First, we identified that the sex-specific differences in developmental strategies were associated with the differential expression of certain key genes, while the within-sex treatment differences were a result of the co-option of addition genes associated with the same developmental pathway. For example, females showed an increased expression of yorkie (a gene associated with increased growth) relative to males, while females reared in silence that grew larger varied in the expression of additional genes associated with increased growth. We found similar associations in patterns of lifespan; males reared in the silent treatment lived the longest and demonstrated the expression of four genes associated with increased lifespan, while females in the silent treatment had the shortest lifespan and expressed four genes associated with decreased lifespan. Males in the calling treatment demonstrated an intermediate lifespan and only expressed a single gene associated with increased lifespan.

Secondly, our results suggest that behavioural phenotypic outcomes are a result from associations of different genes interacting as modules. Whilst interaction of genes in modulating development is well known, our study confirms that this also occurs in an ecological, social and behavioural setting. For example, both males and females that increased their reproductive output had increases in genes associated with that trait and energy producing pathways. Males reared in silence called more, expressed a gene associated with greater courtship, and expressed two genes associated with the storage of greater energy reserves and the production of more efficient muscles. Females reared in the calling treatment produced more eggs, expressed six genes associated with germline maintenance and greater reproductive capacity, and expressed four genes associated with energy producing pathways. Our results thus suggest that the moderation of phenotypes in continuously varying species may be associated with the expression of additional genes, rather than dose-dependence of a smaller subset of genes. This, however, needs to be confirmed in future studies.

Because of the ecological and evolutionary understanding of the various phenotypes in T. commodus, we provide unique evidence for the focus of these genes in future evolutionary and ecological studies. Our results also allow researchers to further explore developmental tactics and the resulting phenotypes in other species from a genomic standpoint as we demonstrate similarities between the T. commodus and Drosophila genes, which likely extends to other species.

\section{Patterns of expression in transcription factors}

Although transcription factors are not well explored outside of model genetic organisms such as Drosophila, identifying relationships between transcription factors and phenotypes in non-model organisms could provide particular insight into important pathways that align with specific life history tactics. To examine the expression patterns in transcription factors, we created a set of transcription factors containing 2,418 transcripts for $T$. commodus through comparison with the D. melanogaster transcription factor library (described in Methods). We used this set to explore differential expression of the transcription factors in each experimental condition. Similar to the differential expression analysis above, we used RSEM to create normalized count tables grouped by age (day 3 vs. day 13) due to the significant difference in gene ontologies used.

Different expression patterns of the transcription factors in individuals of each age group were clustered into $5 \times 5$ grids by self-organized maps (SOMs; Fig. 5), as these best visually described gene clustering. For day 3 , we next selected the two cells that had opposite expression patterns between treatments (Cells 3 and 18) and between sexes (Cells 10 and 16) (Fig. 5a). Cell 3 had 


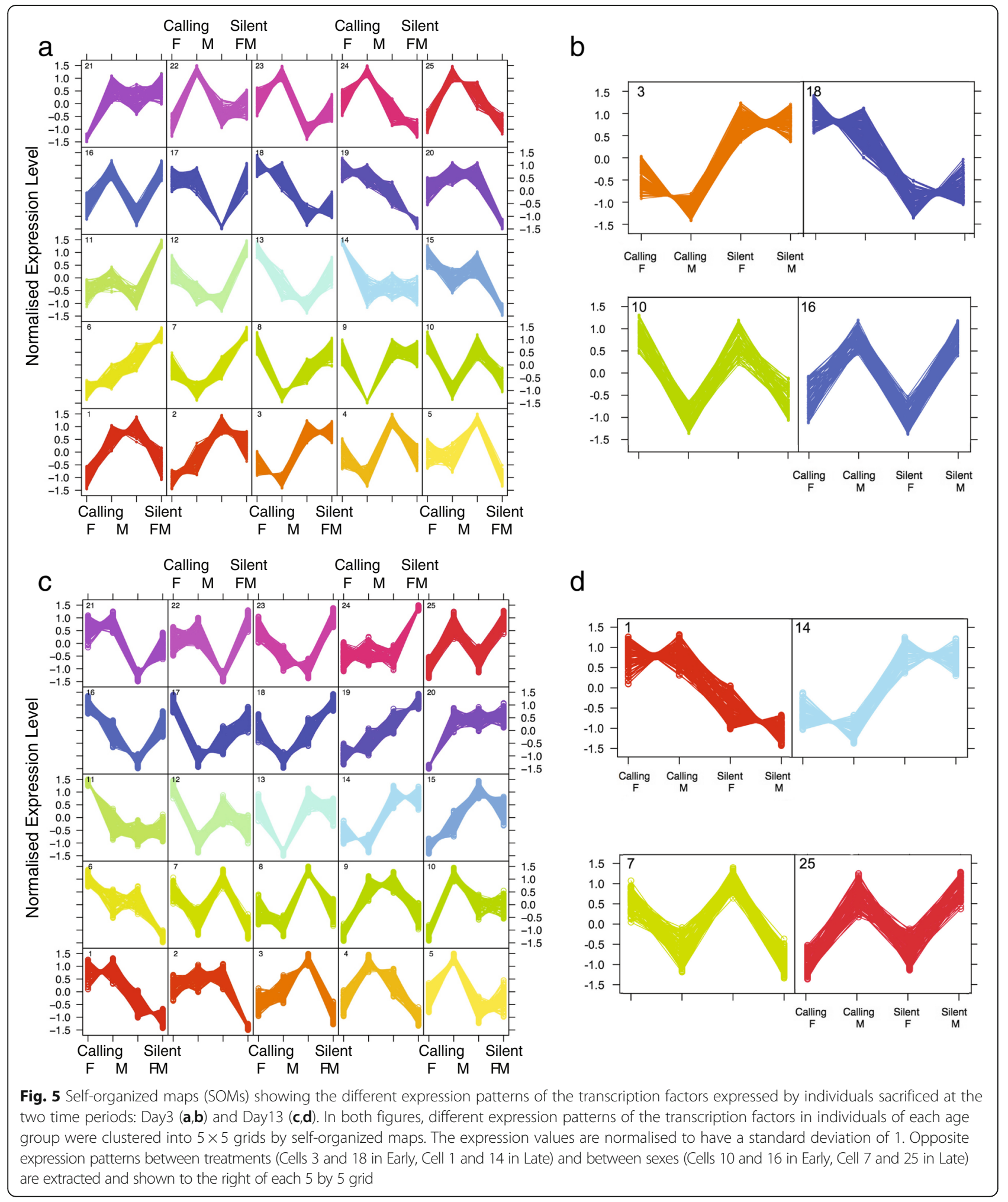

a total of 126 transcription factors overexpressed in the silent treatment when compared to the calling treatment, while Cell 18 had 116 transcription factors overexpressed in the calling treatment when compared to the silent treatment. Cell 10 had a total of 89 transcription factors overexpressed in females when compared to males, while Cell 16 had 87 transcription factors overexpressed in males when compared to females. 
We performed the same analysis for day 13 and found that Cell 14 had a total of 81 transcription factors overexpressed in the silent treatment when compared to the calling treatment, while Cell 1 had 93 transcription factors overexpressed in the calling treatment when compared to the silent treatment (Fig. 5b). In the sex comparison, Cell 7 had 92 transcription factors overexpressed in females when compared to males, and Cell 25 had 95 transcription factors overexpressed in males when compared to females.

We next performed a similar analysis as in the exploration of unique genes (above) to examine unique transcription factor expression, but limited our exploration to between treatments or sexes as the SOMs could not be created for a $2 \times 2 \times 2$ interaction. We found a total of 31 transcription factors associated with the traits of interest in our study, 26 of which only appeared in a single sex or treatment. We discuss the two temporal periods together below.

\section{Treatment differences in transcription factor expression}

Individuals reared in the silent treatment had a slower development rate compared to individuals in the calling treatment (although this was driven by females; Fig. 1a). Despite this, individuals from the silent treatment expressed several transcription factors positively associated with growth and maturation, although each affected growth indirectly through interactions with juvenile hormone in some manner. Foxo is a transcription factor that regulates growth and the specific role it plays depends on the other genes that it interacts with [90]. Ecdysone-induced protein $75 \mathrm{~B}$ is necessary for proper molting to occur [91], and broad is involved in ensuring proper expression of let-7, a small regulatory RNA that promotes transition from larva to adult [92]. Ultraspiracle is involved in tissue specific control of hormonal regulation [93].

Individuals from the calling treatment had a faster development rate, again largely driven by females (Fig. 1a). In contrast to individuals reared in the silent treatment, the two transcription factors directly play roles in development. The first, Topoisomerase $3 \alpha$, is not only required for growth, but it is also involved in the more rapid growth necessary during compensatory growth [94]. The second transcription factor, 14-3-3e, acts as a modulator of Foxo [95] an important transcription factor that regulates growth [90].

\section{Sex-differences in transcription factor expression}

Females had a higher growth rate relative to males in our experiment. Females also showed an increased expression of Pdp-1, a transcription factor associated with growth [96]. Pdp-1 is also associated with increased deposition of fat, which may explain why females are heavier and may also be necessary for the energetic requirements of egg production. CG8578 is also associated with increased muscle development, but little is known about its actual function [97]. Females also demonstrated an overexpression of MTF-1, a transcription factor associated with increased lifespan as it maintains metal homeostasis [98]. Of the 4 transcription factors overexpressed by males, none seem to specifically relate to the traits studied here.

\section{Summary}

In our transcription factor analysis, we could not individually examine each sex within each treatment as in the overall gene expression results, thus resulting in weaker associations as transcription factors and the traits of interest. Nonetheless, we did see differences in the roles the genes played from simply being a part of the developmental process in individuals reared in the silent, to playing a regulating role in individuals reared in the calling treatment. Our results thus once again highlight the interactive role of genes in moderating individual development, and more importantly, place them within an ecological and social context. In each treatment or sex comparison, we found increased expression of several transcription factors that function in single or multiple functional processes. The fact that multiple transcription factors involved in similar roles increased in expression demonstrates redundancy within the developmental system, and could signify that genes are interacting with one another to result in an increased effect; this is similar to our genome wide transcriptome results above.

\section{Conclusions}

The juvenile environment provides numerous cues regarding the potential challenges that individuals should encounter at maturity. If reliable enough [99], the presence of these cues should allow individuals to modify their investment patterns, thereby altering their developmental trajectory $[1,4,5]$. Despite having a strong understanding of the various ecological factors that trigger plastic developmental strategies, we have a poor understanding of the underlying genetic changes that accompany these developmental shifts. Are continuously distributed phenotypes a consequence of a dose-dependent reaction of particular genes? Or are phenotypic differences a consequence of the expression of additional genes with similar function? Understanding the underlying mechanistic patterns can help us understand the evolution of such plasticity, the extent of the potential constraints of plasticity, and the existence of sex-differences in developmental patterns.

Our study provides insight into each of these questions as we demonstrate that the socially-induced developmental plasticity of the Australian black field cricket (T. commodus) is associated with changes in the expression of suites of genes, including key transcription factors, associated with life-history, behavioural, and morphological traits that are under strong natural and sexual selection in this species. Additionally, because we looked at a specific subset of genes rather than simply gene ontology clustering, we provide numerous candidate genes and transcription factors whose roles were delineated through mutations and knock-outs using laboratory model species. Our results hint towards an 
association between gene function and phenotype as more extreme phenotypes were associated with the expression of a larger number of genes associated with that phenotype. This was seen in different trait domains as size, development time, egg output, courtship, and lifespan. Our results thus suggests that continuous phenotypes are a consequence of many interacting genes that together act as a dose-dependent regulator of a phenotype. Alternatively, the use of different genes may have the benefit of ensuring redundancy in developmental programs. Future studies examining whether developmental systems have finer control as a function of this redundancy and whether greater redundancy is common in species with continuous, rather than discrete phenotypes would provide greater insight into the evolution of plasticity.

We demonstrate that the different developmental tactics used by males and females in response to the same acoustic cues may be controlled by different subsets of genes, providing some insight to the sex-specific developmental strategies. For example, female $T$. commodus are larger and develop more quickly [100] and this is associated with the expression of a greater number of genes associated with larger size and faster development. Additionally, our lifespan differences between the sexes and the negative association between the genes expressed and lifespan specifically in females may be an example of the costs associated with particular developmental strategies. Although our study did not specifically examine sexual conflict in developmental strategies, further studies using the candidate genes outlined in this study to explicitly explore sex-specific trade-offs in development and behaviour as well as sexual conflict in mating [e.g., 20] and developmental strategies may prove fruitful.

Our results also show that in cases where expressing specific phenotypes is costly, such as increases in reproductive output, the genes associated with the trait of interest are coupled with increased expression in energy producing pathways. This suggests a second level of interaction between genes and the reliance between gene pathways. The relationship between such pathways may provide insight into questions of condition dependence [101, 102] - does increased investment in energy producing pathways reduce the cost of trait expression? Greater investment in underlying physiological systems may help animals to overcome certain costs of different life-history trajectories such that the costs are not readily apparent with the measure of a specific subset of phenotypic traits [103, 104]. This may make the identification of the costs of phenotypic plasticity more difficult to uncover $[105,106]$.

Our results are also surprising for a different reason. Despite finding strong developmental differences between the sexes and treatments, we did not find differential expression in some of the genes and pathways that are normally expected from such developmental shifts. For example, changes in the expression of heat shock proteins are often linked to increases in stress within the internal and external environment [107]. Relevant to our study, increased density is known to increase the expression of heat shock proteins, and this can subsequently increase longevity [108]. We saw no changes in heat shock proteins in our study, suggesting that auditory cues of density may not trigger the expression of heat shock proteins in the same way as physical cues of density. We also expected to see expression differences in the neuroendocrine axis $[109,50,110]$ due to the size difference in females from the different treatments. We did not see a difference in dopamine expression, which is known to co-occur with juvenile hormone [111], and would therefore affect size at maturity. We also did not see expression differences in prothoracicotropic hormone (PTTH) which was surprising given that PTTH is secreted in the brain and begins the downstream expression of other hormones associated with molting. This, however, may be a consequence of the tight timing of PTTH with molting $[109,50]$ such that our sacrificing of individuals did not align with this timing. Alternatively, the differences associated with development in response to social cues may be cumulative compared to the relatively more abrupt physiological changes occurring during molting. Despite finding associations between lifespan and gene expression in our study, we did not see differences in the expression of methuselah, a gene that is strongly associated with lifespan in Drosophila [112]. This may be because although methuselah is associated with lifespan differences in the lab, such associations may not exist in wild populations despite natural variation in methuselah expression [113].

Overall, our results speak to the importance of examining expression patterns of 'normal' wild caught individuals as this demonstrates the interactive importance of genes in different phenotypic outcomes and that associations may vary between laboratory and natural populations due to the variation in selection. Our results also highlight the importance of examining the genomic underpinning of plasticity in response to various triggering factors as different environments and triggers may require the use of different developmental and physiological pathways. We also demonstrate that the genetic factors underlying developmental patterns can be uncovered in continuously variable species when a strong evolutionary and ecological understanding is coupled with a genomic approach [114]. Such an understanding cannot be gained through using laboratory strains of knock-outs and mutants alone [115]. This will hopefully encourage future genomic studies on non-model organisms.

\section{Additional files}

Additional file 1: Figure S1. Expression patterns (log2-transformed, median centered) of the two clusters showing significant differences in gene expression between early (Day 3) and late (Day 13). The blue line indicates the mean-centered expression patterns of each cluster. The grey lines indicate individual expression patterns of each gene. Figure S2. 
Flowchart showing the workflow for the transcriptome assembly, evaluation and annotation. Table S1. The percentage of reads mapped to the three transcriptomes assembled by different assemblers. Table S2. Statistics of the assembled transcriptomes by different assemblers and redundancy removal steps. (DOCX $1765 \mathrm{~kb})$

Additional file 2: Supplementary Materials and Results. (DOCX $80 \mathrm{~kb}$ ) Additional file 3: Supplementary Excel file (XLSX 29 kb)

\section{Acknowledgements}

The authors would like to thank Heather Try for all her help rearing crickets, Anna Kopps for her help in cDNA extraction, the Ramaciotti Centre for Genomics for running the samples and discussions of results, and Matt Hall for discussions about gene expression.

\section{Funding}

This research was funded by an ARC Discovery Project (DP120101502) and ARC DECRA Fellowship (DE120100214) to MMK and New South Wales State Government Science Leveraging Fund (SLF) and by the University of New South Wales to MRW. The funders had no role in study design, data collection and analysis, decision to publish, or preparation of the manuscript.

\section{Availability of data and materials}

This Transcriptome Shotgun Assembly project has been deposited at DDBJ/ EMBL/GenBank under the accession GBHB00000000. The version described in this paper is the first version, GBHB01000000. Raw reads of the twenty-four samples were deposited in SRA under accession numbers of: SAMN02863001, SAMN02863002, SAMN02863003, SAMN02863004, SAMN02863005, SAMN02863006, SAMN02863007, SAMN02863008, SAMN02863009, SAMN02863010, SAMN02863011, SAMN02863012, SAMN02863013, SAMN02863014, SAMN02863015, SAMN02863016, SAMN02863017, SAMN02863018, SAMN02863019, SAMN02863020, SAMN02863021, SAMN02863022, SAMN02863023, SAMN02863024.

\section{Authors' contributions}

MMK, ZC, and MRW made substantial contributions to conception and design; MMK and ZC acquired, interpreted and analyzed data; MMK, ZC, and MRW wrote the manuscript. All authors read and approved the final manuscript.

\section{Competing interest}

The authors declare that they have no competing interests.

\section{Author details}

${ }^{1}$ Ecology \& Evolution Research Centre, School of Biological, Earth and Environmental Sciences, UNSW, Sydney, Australia. ${ }^{2}$ Systems Biology Initiative, UNSW, Sydney, Australia. ${ }^{3}$ School of Biotechnology and Biomolecular Sciences, UNSW, Sydney, Australia.

\section{Received: 6 April 2016 Accepted: 23 September 2016}

Published online: 24 October 2016

\section{References}

1. West-Eberhard MJ. Developmental plasticity and evolution. New York: Oxford University Press; 2003.

2. Loman J. Early metamorphosis in common frog Rana temporaria tadpoles at risk of drying: An experimental demonstration. Amphibia-Reptilia. 1999;20:421-30.

3. Kasumovic MM: The multidimensional consequences of the juvenile environment: towards an integrative view of the adult phenotype Anim Behav 2013:In Press.

4. Pigliucci M. Phenotypic plasticity: beyond nature and nurture. Baltimore, MD: John Hopkins University Press; 2001.

5. Kasumovic MM, Brooks R. It's all who you know: The evolution of sociallycued anticipatory plasticity as a mating strategy. Q Rev Biol. 2011;86:181-97.

6. Adler FR, Harvell CD. Inducible Defenses, Phenotypic Variability and Biotic Environments. Trends Ecol Evol. 1990;5(12):407-10.

7. Nylin S, Gotthard K. Plasticity in life-history traits. Annu Rev Entomol. 1998;43:63-83.
8. Scheiner SM: The genetics of phenotypic plasticity. XII. Temporal and spatial heterogeneity. Ecology and Evolution 2013:n/a-n/a.

9. Laurila A, Karttunen S, Merilä J. Adaptive phenotypic plasticity and genetics of larval life histories in two Rana temporaria populations. Evolution. 2002;56(3):617-27

10. Johansson F, Stoks R, Rowe L, De Block M. Life history plasticity in a damselfly: Effects of combined time and biotic constraints. Ecology. 2001;82(7):1857-69.

11. Relyea RA, Auld JR. Predator- and competitor-induced plasticity: How changes in foraging morphology affect phenotypic trade-offs. Ecology. 2005;86:1723-9.

12. de Jong G. Evolution of phenotypic plasticity: patterns of plasticity and the emergence of ecotypes. New Phytol. 2005;166:101-18.

13. Scheiner SM. Genetics and evolution of phenotypic plasticity. Ann Rev Ecol Syst. 1993;24:35-68.

14. Van Tienderen PH. Generalists, specialists, and the evolution of phenotypic plasticity in sympatric populations of distinct species. Evolution. 1997;51:1372-80.

15. Kijimoto T, Snell-Rood EC, Pespeni MH, Rocha G, Kafadar K, Moczek AP: The nutritionally responsive transcriptome of the polyphenic beetle Onthophagus taurus and the importance of sexual dimorphism and body region. Proceedings B: Biological Sciences. 2014;281:20142084-20142084. http://rspb.royalsocietypublishing.org/content/281/1797/20142084.

16. Adams HA, Southey BR, Robinson GE, Rodriguez-Zas SL. Meta-analysis of genome-wide expression patterns associated with behavioral maturation in honey bees. BMC Genomics. 2008:9(1):503.

17. Moczek AP. The nature of nurture and the future of evodevo: Towards a theory of developmental evolution. Integr Comp Biol. 2012;52:108-19.

18. Bentsen $C L$, Hunt J, Jennions MD, Brooks R. Complex multivariate sexual selection on male acoustic signaling in a wild population of Teleogryllus commodus. Am Nat. 2006;167(4):E102-16.

19. Bussière LF, Hunt J, Jennions MD, Brooks R. Sexual conflict and cryptic female choice in the black field cricket. Teleogryllus commodus Evolution. 2006:59:871-80

20. Hall MD, Bussière LF, Hunt J, Brooks R. Experimental evidence that sexual conflict influences the opportunity, form and intensity of sexual selection. Evolution. 2008;62(9):2305-15.

21. Hunt J, Brooks R, Jennions MD, Smith MJ, Bentsen CL, Bussière LF. Highquality male field crickets invest heavily in sexual display but die young. Nature. 2004:432(7020):1024-7.

22. Zajitschek F, Hunt J, Jennions MD, Hall MD, Brooks RC. Effects of juvenile and adult diet on ageing and reproductive effort of male and female black field crickets. Teleogryllus commodus Func Ecol. 2009;23(3):602-11.

23. Kasumovic MM, Hall MD, Try H, Brooks R. The importance of listening: allocation shifts in response to the juvenile acoustic environment. J Evol Biol. 2011;24:1325-34.

24. Kasumovic MM, Hall MD, Brooks R. The juvenile social environment introduces variation in the choice and expression of sexually selected traits. Ecol Evol. 2012;2:1036-47.

25. Tamayo P, Slonim D, Mesirov J, Zhu Q, Kitareewan S, Dmitrovsky E, Lander ES, Golub TR. Interpreting patterns of gene expression with self-organizing maps: Methods and application to hematopoietic differentiation. Proc Natl Acad Sci U S A. 1999:96:2907-12.

26. Hammonds AS, Bristow CA, Fisher WW, Weiszmann R, Wu S, Hartenstein V, Kellis M, Yu B, Frise E, Celniker SE. Spatial expression of transcription factors in Drosophila embryonic organ development. Genome Biol. 2013;14:R140.

27. Bailey NW, Gray B, Zuk M. Acoustic experience shapes alternative mating tactics and reproductive investment in male field crickets. Curr Biol. 2010:20:845-9.

28. Bailey NW, Zuk M. Acoustic experience shapes female choice in field crickets. Proc R Soc B. 2008:275:2645-50.

29. Lailvaux SP, Hall MD, Brooks R. Performance is no proxy for genetic quality: Trade-offs between locomotion, attractiveness, and life history in crickets. Ecology. 2010;91:1530-7.

30. FastQC A quality control tool for high throughput sequence data [http://www.bioinformatics.babraham.ac.uk/projects/fastqc/]

31. Martin M. Cutadapt removes adapter sequences from high-throughput sequencing reads. EMBnetjournal. 2011;17:10-2.

32. Cox MP, Peterson DA, Biggs PJ. SolexaQA: At-a-glance quality assessment of Illumina second-generation sequencing data. BMC bioinformatics. 2010;11:485. 
33. Birol I, Jackman SD, Nielsen CB, Qian JQ, Varhol R, Stazyk G, Morin RD, Zhao $Y$, Hirst M, Schein JE, et al. De novo transcriptome assembly with ABySS. Bioinformatics. 2009;25:2872-7.

34. Schulz MH, Zerbino DR, Vingron M, Birney E. Oases: robust de novo RNA-seq assembly across the dynamic range of expression levels. Bioinformatics. 2012;28:1086-92.

35. Grabherr MG, Haas BJ, Yassour M, Levin JZ, Thompson DA, Amit I, Adiconis X, Fan L, Raychowdhury R, Zeng Q, et al. Full-length transcriptome assembly from RNA-seq data without a reference genome. Nat Biotechnol. 2011;29:644-52.

36. Langmead B, Salzberg SL. Fast gapped-read alignment with Bowtie 2 . Nat Methods. 2012;9(4):357-9.

37. Li H, Handsaker B, Wysoker A, Fennell T, Ruan J, Homer N, Marth G, Abecasis G, Durbin R. The Sequence Alignment/Map format and SAMtools. Bioinformatics. 2009;25(16):2078-9.

38. Bailey NW, Veltsos P, Tan Y, Millar AH, Ritchie MG, Simmons LW. TissueSpecific Transcriptomics in the Field Cricket Teleogryllus oceanicus. Genes Genomes Genetics. 2013;3:225-30.

39. Pearson WR: An Introduction to Sequence Similarity ("Homology") Searching. Current Protocols in Bioinformatics. 2013;42:3.1:3.1.1-3.1.8. http:// onlinelibrary.wiley.com/doi/10.1002/0471250953.bi0301s42/ abstract? userlsAuthenticated=false\&deniedAccessCustomisedMessage $=$.

40. Marygold SJ, Leyland PC, Seal RL, Goodman JL, Thurmond J, Strelets VB, Wilson RJ. FlyBase: improvements to the bibliography. Nucleic Acids Res. 2013;41(Database issue):D751-757.

41. Ye J, Fang L, Zheng H, Zhang Y, Chen J, Zhang Z, Wang J, Li S, Li R, Bolund L, et al. WEGO: a web tool for plotting GO annotations. Nucleic Acids Res. 2006;34(Web Server issue):W293-297.

42. Kanehisa M, Goto S, Kawashima S, Okuno Y, Hattori M. The KEGG resource for deciphering the genome. Nucleic Acids Res. 2004;32(Database issue):D277-280.

43. Li B, Dewey CN. RSEM: accurate transcript quantification from RNA-Seq data with or without a reference genome. BMC bioinformatics. 2011;12:323.

44. Robinson MD, McCarthy DJ, Smyth GK. edgeR: a Bioconductor package for differential expression analysis of digital gene expression data. Bioinformatics. 2010;26(1):139-40.

45. da Huang W, Sherman BT, Lempicki RA. Systematic and integrative analysis of large gene lists using DAVID bioinformatics resources. Nat Protoc 2009:4(1):44-57.

46. Adryan B, Teichmann SA. FlyTF: a systematic review of site-specific transcription factors in the fruit fly Drosophila melanogaster. Bioinformatics. 2006;22:1532-3.

47. Gentleman R, Carey V, Huber W, Hahne F: genefilter: genefilter: methods for filtering genes from high-throughput experiments. $R$ package version 1.50.0.

48. Melssen WJ, Wehrens R, Buydens LMC. Supervised Kohonen networks for classification problems. Chemom Intell Lab Syst. 2006;83:99-113.

49. Kasumovic MM, Hall MD, Try H, Brooks R. Socially cued developmental plasticity affects condition-dependent trait expression. Behav Ecol. 2012;24:429-34

50. Nijhout HF. Insect Hormones. Princeton, New Jersey: Princeton University Press; 1994.

51. Simonsen A, Cumming RC, Lindmo K, Galaviz V, Cheng S, Rusten TE, Finley KD. Genetic modifiers of the Drosophila blue cheese gene link defects in lysosomal transport with decreased life span and altered ubiquitinatedprotein profiles. Genetics. 2007;176(2):1283-97.

52. Wang $M C$, Bohmann $D$, Jasper $H$. JNK signaling confers tolerance to oxidative stress and extends lifespan in Drosophila. Dev Cell. 2003;5(5):811-6.

53. Wu H, Wang MC, Bohmann D. JNK protects Drosophila from oxidative stress by trancriptionally activating autophagy. Mech Dev. 2009;126(8-9):624-37.

54. Libert S, Chao Y, Zwiener J, Pletcher SD. Realized immune response is enhanced in long-lived puc and chico mutants but is unaffected by dietary restriction. Mol Immunol. 2008;45(3):810-7.

55. Bosch M, Serras F, Martín-Blanco E, Baguñà J. JNK signaling pathway required for wound healing in regenerating Drosophila wing imaginal discs. Dev Biol. 2005:280(1):73-86.

56. Rämet M, Lanot R, Zachary D, Manfruelli P. JNK signaling pathway is required for efficient wound healing in Drosophila. Dev Biol. 2002;241(1):145-56.

57. Vrailas-Mortimer A, del Rivero T, Mukherjee S, Nag S, Gaitanidis A, Kadas D, Consoulas C, Duttaroy A, Sanyal S. A muscle-specific p38 MAPK/Mef2/ MnSOD pathway regulates stress, motor function, and life span in Drosophila. Dev Cell. 2011;21(4):783-95.
58. Ryabinina OP, Subbian E, lordanov MS. D-MEKK1, the Drosophila orthologue of mammalian MEKK4/MTK1, and Hemipterous/D-MKK7 mediate the activation of D-JNK by cadmium and arsenite in Schneider cells. BMC Cell Biol. 2006;7:7.

59. Kishita Y, Tsuda M, Aigaki T. Impaired fatty acid oxidation in a Drosophila model of mitochondrial trifunctional protein (MTP) deficiency. Biochem Biophys Res Commun. 2012;419(2):344-9.

60. Campos I, Geiger JA, Santos AC, Carlos V, Jacinto A. Genetic screen in Drosophila melanogaster uncovers a novel set of genes required for embryonic epithelial repair. Genetics. 2010;184(1):129-40.

61. Carhan A, Allen F, Armstrong JD, Hortsch M, Goodwin SF, O'Dell KMC. Female receptivity phenotype of icebox mutants caused by a mutation in the L1-type cell adhesion molecule neuroglian. Genes Brain Behav. 2005:4(8):449-65.

62. Merritt TJS, Sezgin E, Zhu C-T, Eanes WF. Triglyceride pools, flight and activity variation at the Gpdh locus in Drosophila melanogaster. Genetics. 2006;172(1):293-304

63. Wilson GF, Wang Z, Chouinard SW, Griffith LC, Ganetzky B. Interaction of the K channel beta subunit, Hyperkinetic, with eag family members. J Biol Chem. 1998;273(11):6389-94.

64. Bashirullah A, Lam G, Yin VP, Thummel CS. dTrf2 is required for transcriptional and developmental responses to ecdysone during Drosophila metamorphosis. Dev Dyn. 2007;236(11):3173-9.

65. Dubrovsky EB, Dubrovskaya VA, Bernardo T, Otte V, Difilippo R, Bryan H. The Drosophila FTZ-F1 Nuclear Receptor Mediates Juvenile Hormone Activation of E75A Gene Expression through an Intracellular Pathway. J Biol Chem. 2011:286(38):33689-700

66. Galloni M, Edgar BA. Cell-autonomous and non-autonomous growthdefective mutants of Drosophila melanogaster. Development. 1999;126(11):2365-75.

67. Nolo R, Morrison CM, Tao C, Zhang X, Halder G. The bantam microRNA is a target of the hippo tumor-suppressor pathway. Curr Biol. 2006;16(19):1895-904.

68. Oh H, Irvine KD. Cooperative Regulation of Growth by Yorkie and Mad through bantam. Dev Cell. 2011;20(1):109-22.

69. Campbell PM, Healy MJ, Oakeshott JG. Characterisation of juvenile hormone esterase in Drosophila melanogaster. Insect Biochem Mol Biol. 1992;22(7):665-77.

70. Ellis LL, Carney GE. Mating alters gene expression patterns in Drosophila melanogaster male heads. BMC Genomics. 2010;11:558.

71. Colombani J, Raisin S, Pantalacci S, Radimerski T, Montagne J, Leopold P. A nutrient sensor mechanism controls Drosophila growth. Cell. 2003;114(6):739-49.

72. Goddeeris MM, Cook-Wiens E, Horton WJ, Wolf H, Stoltzfus JR, Borrusch M, Grotewiel MS. Delayed behavioural aging and altered mortality in Drosophila beta integrin mutants. Aging Cell. 2003;2(5):257-64.

73. Bai H, Kang P, Hernandez AM, Tatar M. Activin Signaling Targeted by Insulin/dFOXO Regulates Aging and Muscle Proteostasis in Drosophila. PLoS Genet. 2013;9(11):e1003941.

74. Mockett RJ, Orr WC, Rahmandar JJ, Benes JJ, Radyuk SN, Klichko VI, Sohal RS Overexpression of Mn-Containing superoxide dismutase in transgenic Drosophila melanogaster. Arch Biochem Biophys. 1999;371(2):260-9.

75. Hull-Thompson J, Muffat J, Sanchez D, Walker DW, Benzer S, Ganfornina MD, Jasper $\mathrm{H}$. Control of metabolic homeostasis by stress signaling is mediated by the lipocalin NLaz. PLoS Genet. 2009:5(4):e1000460.

76. Maklakov AA, Hall MD, Simpson SJ, Dessman J, Clissold FJ, Zaitschek F, Lailvauz SP, Raubenheimer D, Bonduriansky R, Brooks RC. Sex differences in nutrient-dependent reproductive ageing. Aging Cell. 2009;8:324-30.

77. Maklakov AA, Simpson SJ, Zajitschek F, Hall MD, Dessman J, Clissold FJ, Raubenheimer D, Bonduriansky R, Brooks R. Sex-specific fitness effects of nutrient intake on reproduction and lifespan. Curr Biol. 2008;18:1062-8.

78. Schupbach T, Wieschaus E. Female sterile mutations on the second chromosome of Drosophila melanogaster. Genetics. 1991;129:1119-36.

79. Gilbert MM, Tipping M, Veraksa A, Moberg KH. A Screen for Conditional Growth Suppressor Genes Identifies the Drosophila Homolog of HD-PTP as a Regulator of the Oncoprotein Yorkie. Dev Cell. 2011;20(5):700-12.

80. Dermaut B, Norga KK, Kania A, Verstreken P, Pan H, Zhou Y, Callaerts P, Bellen HJ. Aberrant lysosomal carbohydrate storage accompanies endocytic defects and neurodegeneration in Drosophila benchwarmer. J Cell Biol. 2005:170(1):127-39.

81. Jagut M, Mihaila-Bodart L, Molla-Herman A, Alin MF, Lepesant JA, Huynh JR. A mosaic genetic screen for genes involved in the early steps of Drosophila oogenesis. G3. 2013;3(3):409-25. 
82. Compagnon J, Gervais L, Roman MS, Chamot-Boeuf S, Guichet A. Interplay between Rab5 and Ptdlns(4,5)P2 controls early endocytosis in the Drosophila germline. J Cell Sci. 2009;122(1):25-35.

83. Perrimon N, Lanjuin A, Arnold C, Noll E. Zygotic lethal mutations with maternal effect phenotypes in Drosophila melanogaster. Genetics. 1996;144(4):1681-92.

84. Jeong K, Kim-Ha J. Precocious expression of Drosophila Rbp9 inhibits ovarian germ cell proliferation. Mol Cells. 2004;18(2):230-6.

85. Oren-Giladi P, Krieger O, Edgar BA, Chamovitz DA, Segal D. Cop9 signalosome subunit 8 (CSN8) is essential for Drosophila development. Genes Cells. 2008;13(3):221-31.

86. Seebacher F, Walter I. Differences in locomotor performance between individuals: importance of parvalbumin, calcium handling and metabolism. J Exp Biol. 2012;215:663-70.

87. Copeland JM, Cho J, Lo T, Hur JH, Bahadorani S, Arabyan T, Rabie J, Soh J, Walker DW. Extension of Drosophila life span by RNAi of the mitochondrial respiratory chain. Curr Biol. 2009;19(19):1591-8.

88. Landis GN, Bhole D, Tower J. A search for doxycycline-dependent mutations that increase Drosophila melanogaster life span identifies the VhaSFD, Sugar baby, filamin, fwd and Cctl genes. Genome Biol. 2003;4(2):R8.

89. Gray B, Simmons LW. Acoustic cues alter perceived sperm competition risk in the field cricket Teleogryllus oceanicus. Behav Ecol. 2013;24(4):982-6.

90. Kramer JM, Davidge JT, Lockyer JM, Staveley BE. Expression of Drosophila FOXO regulates growth and can phenocopy starvation. BMC Dev Biol. 2003;3(1):5.

91. Bialecki M, Shilton A, Fichtenberg C, Segraves WA, Thummel CS. Loss of the ecdysteroid-inducible E75A orphan nuclear receptor uncouples molting from metamorphosis in Drosophila. Dev Cell. 2002;3(2):209-20.

92. Sempere LF, Dubrovsky EB, Dubrovskaya VA, Berger EM, Ambros V. The expression of the let-7 small regulatory RNA is controlled by ecdysone during metamorphosis in Drosophila melanogaster. Dev Biol. 2002;244(1):170-9.

93. Beck Y, Delaporte C, Moras D, Richards G, Billas IM. The ligand-binding domains of the three RXR-USP nuclear receptor types support distinct tissue and ligand specific hormonal responses in transgenic Drosophila. Dev Biol. 2009:330(1):1-11.

94. Gerhold AR, Richter DJ, Yu AS, Hariharan IK. Identification and characterization of genes required for compensatory growth in Drosophila. Genetics. 2011;189(4):1309-26.

95. Nielsen MD, Luo X, Biteau B, Syverson K, Jasper H. 14-3-3 Epsilon antagonizes FoxO to control growth, apoptosis and longevity in Drosophila. Aging Cell. 2008;7(5):688-99.

96. Reddy KL, Rovani MK, Wohlwill A, Katzen A, Storti RV. The Drosophila Par domain protein I gene, $\mathrm{Pdp} 1$, is a regulator of larval growth, mitosis and endoreplication. Dev Biol. 2006;289(1):100-14.

97. Schnorrer F, Schönbauer C, Langer CC, Dietzl G, Novatchkova M, Schernhuber K, Fellner M, Azaryan A, Radolf M, Stark A, et al. Systematic genetic analysis of muscle morphogenesis and function in Drosophila. Nature. 2010;464(7286):287-91.

98. Selvaraj A, Balamurugan K, Yepiskoposyan H, Zhou H, Egli D, Georgiev O, Thiele DJ, Schaffner W. Metal-responsive transcription factor (MTF-1) handles both extremes, copper load and copper starvation, by activating different genes. Genes Dev. 2005;19(8):891-6.

99. Lively CM. Canalization versus developmental conversion in a spatiallyvariable environment. Am Nat. 1986;128(4):561-72.

100. Zajitschek F, Bonduriansky R, Zajitschek SR, Brooks RC. Sexual dimorphism in life history: age, survival, and reproduction in male and female field crickets Teleogryllus commodus under seminatural conditions. Am Nat. 2009;173(6):792-802.

101. Rowe L, Houle D. The lek paradox and the capture of genetic variance by condition dependent traits. Proc Roy Soc B. 1996;263(1375):1415-21.

102. Cotton S, Fowler K, Pomiankowski A. Do sexual ornaments demonstrate heightened condition-dependent expression as predicted by the handicap hypothesis? Proc Roy Soc London Ser B. 2004;271:771-83.

103. Relyea RA, Auld JR. Having the guts to compete: how intestinal plasticity explains costs of inducible defences. Ecol Lett. 2004;7:869-75.

104. Relyea RA. Local population differences in phenotypic plasticity: predator induced changes in wood frog tadpoles. Ecol Monogr. 2002;72:77-93.

105. Snell-Rood EC. An overview of the evolutionary causes and consequences of behavioural plasticity. Anim Behav. 2013:85:1004-11.
106. Snell-Rood EC, Van Dyken JD, Cruickshank T, Wade MJ, Moczek AP. Toward a population genetic framework of developmental evolution: the costs, limits, and consequences of phenotypic plasticity. Bioessays. 2010;32(1):71-81.

107. Sørensen JG, Kristensen TN, Loeschcke V. The evolutionary and ecological role of heat shock proteins. Ecol Lett. 2003;6:1025-37.

108. Sørensen JG, Loeschcke V. Larval crowding in Drosophila melanogaster induces Hsp70 expression, and leads to increased adult longevity and adult thermal stress resistance. J Insect Physiol. 2001;47:1301-1307.

109. Callier V, Nijhout HF. Body size determination in insects: a review and synthesis of size- and brain-dependent and independent mechanisms. Biol Rev. 2013:88(4):944-54.

110. VaN Callier HF. Body size determination in insects: a review and synthesis of size- and brain-dependent and independent mechanisms. Biological Rev. 2013;88:944-54.

111. Gruntenko NE, Karpova EK, Alekseev AA, Chentsova NA, Saprykina ZV, Bownes M, Rauschenbach IY. Effects of dopamine on juvenile hormone metabolism and fitness in Drosophila virilis. J Insect Physiol. 2005;51:959-68.

112. Gimenez LED, Ghildyal P, Fischer KE, Hu H, Ja WW, Eaton BA, Wu Y, Austad SN, Ranjan R. Modulation of methuselah expression targeted to Drosophila insulin-producing cells extends life and enhances oxidative stress resistance. Aging Cell. 2013;12:121-9.

113. Sgrò CM, Van Heerwaarden B, Kellermann V, Wee CW, Hoffmann AA, Lee SF. Complexity of the genetic basis of ageing in nature revealed by a clinal study of lifespan and methuselah, a gene for ageing, in Drosophila from eastern Australia. Mol Ecol. 2013;22:3539-51.

114. Zuk M, Ballenger SL. Behavioral ecology and genomics: new directions, or just a more detailed map? Behav Ecol. 2014;25:1277-82.

115. Zuk M, Garcia-Gonzalez F, Herberstein ME, Simmons LW. Model systems, taxonomic bias, and sexual selection: beyond Drosophila. Annu Rev Entomol. 2014;59:321-38.

\section{Submit your next manuscript to BioMed Central and we will help you at every step:}

- We accept pre-submission inquiries

- Our selector tool helps you to find the most relevant journal

- We provide round the clock customer support

- Convenient online submission

- Thorough peer review

- Inclusion in PubMed and all major indexing services

- Maximum visibility for your research

Submit your manuscript at www.biomedcentral.com/submit
C) Biomed Central 